\title{
New structurally unstable families of planar vector fields
}

\author{
Nataliya Goncharuk Yury G. Kudryashov \\ Nikita Solodovnikov
}

March 11, 2022

\begin{abstract}
We study global bifurcations in generic 3-parameter families of vector fields on $S^{2}$. In the recent article (Ilyashenko, Kudryashov, and Schurov, 2018), the authors show that 3-parameter unfoldings of vector fields with the polycycle "tears of the heart" are structurally unstable. We consider 3-parameter unfoldings of vector fields with separatrix graphs "ears" and "glasses", and prove that these families are structurally unstable as well.

We also study in more details the classical bifurcation of a saddle loop, and use it as a building block in our main example.
\end{abstract}

\section{Introduction}

It is well-known $1,2,3,5,21,22,23$ that a generic vector field on $S^{2}$ is structurally stable. Sotomayor 28 classified so-called quasi-generic vector fields on the sphere, i.e. vector fields generic inside the class of structurally unstable vector fields. Quasi-generic vector fields have exactly one of the following degeneracies:

1. a quasi-generic singular point, i.e. a saddle-node, or a composed focus;

2. a separatrix connection between different saddles;

3. a semi-stable limit cycle of multiplicity 2 ;

4. a separatrix loop.

He also described bifurcations that occur in 1-parametric unfoldings of quasi-generic vector fields near the "interesting" parts of their phase portraits. In particular, this description implies that generic 1-parameter unfoldings are structurally stable, if one restricts them to small neighbourhoods of their degeneracies listed above. Bifurcations of this type are called local, if the degeneracy is a singular point, and non-local, if it is a polycycle or a degenerate limit cycle.

However, the corresponding global bifurcations can be more complicated. In [20], Malta and Palis described global bifurcations in the two most interesting cases 3 and 4 , 
stated their classification up to the topological equivalence (this and other equivalence relations are defined in Definition 3), and sketched the proof of this classification. In case 3 of a semi-stable limit cycle this classification has numerical moduli, and in case 4 of a separatrix loop this classification has no moduli. Later Dumortier and Roussarie 9 proved that in the latter case numerical moduli appear, if one imposes additional regularity assumptions on the conjugating homeomorphism. In Sec. 3 we prove a modified version of this result for the weak topological equivalence with similar modifications.

For families with more parameters, classification up to the topological equivalence has functional moduli. This was proved by Roussarie [26] for non-local bifurcations in a 3-parameter family, and this example can be easily modified to produce functional invariants for global bifurcations in a 2-parameter family, see [12] for details and another way to obtain functional invariants in the latter case.

In 1986, Arnold 4 conjectured ${ }^{1}$ that these moduli, both numerical and functional, appear because the topological equivalence relation is too strict. He proposed a much less restrictive notion of weak topological equivalence, and formulated 6 conjectures about global bifurcations of finite parameter families of vector fields on $S^{2}$. One of them states that a generic family of this type is weakly structurally stable. Though most of these conjectures turned out to be wrong, they influenced research in this area for decades.

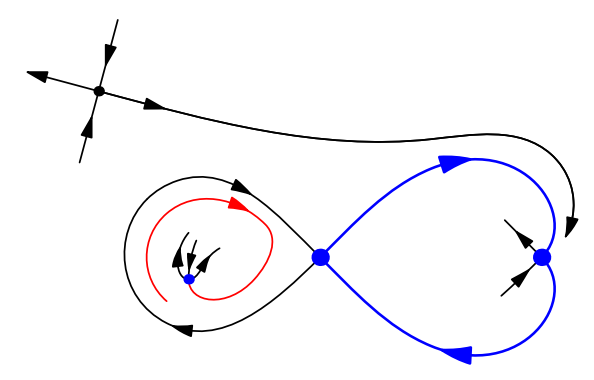

Figure 1: A vector field with polycycle "tears of the heart"

Recently Ilyashenko, Kudryashov, and Schurov [16 came up with an open set in the space of 3-parameter families of vector fields on the sphere such that all families in this set are structurally unstable. These families are unfoldings of vector feilds with "tears of the heart" polycycle, see Fig. 1. For technical reasons, this result was proved for the moderate equivalence relation that is stronger than the weak equivalence but is weaker than the original notion of topological equivalence. Later, a modification of the construction used in 16 was used in 18 to construct metrically generic 3-parameter families of vector fields with arbitrafily many numerical invariants.

This result motivated a few series of questions about generic families of vector fields on the sphere.

1-parameter families: fully classify them up to the weak equivalence.

This was accomplished in $[13,17,29]$. In particular, generic 1-parameter families of vector fields on the sphere are structurally stable.

\footnotetext{
${ }^{1}$ According to Yu. Ilyashenko, this section of the book was written entirely by V. Arnold.
} 


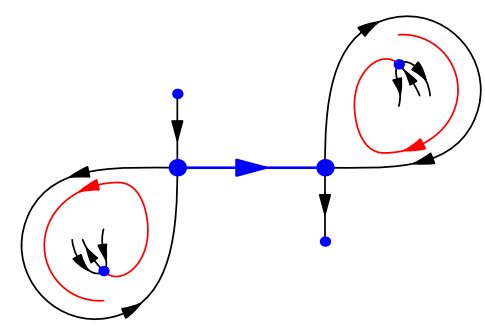

(a) "Ears"

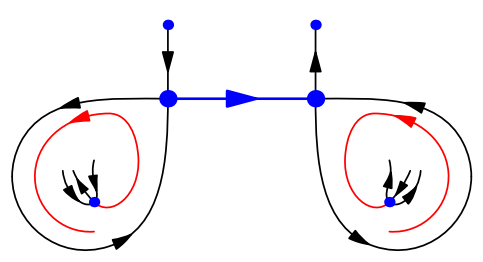

(b) "Glasses"

Figure 2: Vector fields with "ears" and "glasses"

2-parameter families: are they generically structurally stable with respect to the weak and the moderate topological equivalences?

We expect that the answer is "yes". Some two-parameter families were studied in $6,19,24,25,27$, but the general question is wide open.

3-parameter families: list all locally generic structurally unstable families.

In this paper we provide two new examples of locally generic structurally unstable 3-parameter families. Namely, generic unfoldings of degenerate vector fields in Fig. 2 are structurally unstable; see Theorem 1 for a precise statement.

finite parameter families: how far can they fall from being structurally stable?

Theorem 2 in [16 provides an example of 6-parameter families with functional invariants. This result was independently improved by Dukov [7] and by the first two authors of the present paper [14, see discussion in Sec. 5.

See also [15] for a more detailed survey of current progress on these and other related questions.

\section{Preliminaries}

\subsection{Families and equivalences ${ }^{2}$}

Denote by Vect the Banach space of $C^{3}$-smooth vector fields on the two-sphere.

Definition 1. Given an open subset $\mathcal{B} \subset \mathbb{R}^{k}$, a map $V: \mathcal{B} \rightarrow$ Vect, $V=\left\{v_{\alpha}\right\}_{\alpha \in \mathcal{B}}$ is called a $k$-parametric family of vector fields. A local family is a germ of a map $V:\left(\mathbb{R}^{k}, 0\right) \rightarrow\left(\right.$ Vect, $\left.v_{0}\right)$. Denote by $\mathcal{V}_{k}$ the Banach space of local families $V=\left\{v_{\alpha}\right\}_{\alpha \in\left(\mathbb{R}^{k}, 0\right)}$ that are $C^{3}$-smooth in $(\alpha, x)$.

To define equivalent families of vector fields, we first introduce an equivalence relation on Vect.

\footnotetext{
${ }^{2}$ Definitions in this section were copied from 16,18 with minor or no modifications.
} 
Definition 2. Two vector fields $v, w \subset$ Vect are called orbitally topologically equivalent, if there exists an orientation preserving homeomorphism $H: S^{2} \rightarrow S^{2}$ that takes phase curves of $v$ to phase curves of $w$, and preserves time orientation.

There are several different "natural" definitions of equivalent families of vector fields.

Definition 3. Consider two local families $V=\left\{v_{\alpha}\right\}_{\alpha \in\left(\mathbb{R}^{k}, 0\right)}$ and $\tilde{V}=\left\{\tilde{v}_{\tilde{\alpha}}\right\}_{\tilde{\alpha} \in\left(\mathbb{R}^{k}, 0\right)}$. A germ of a map

$$
H:\left(\mathbb{R}^{k} \times S^{2},\{0\} \times S^{2}\right) \rightarrow\left(\mathbb{R}^{k} \times S^{2},\{0\} \times S^{2}\right), \quad H(\alpha, x)=\left(h(\alpha), H_{\alpha}(x)\right)
$$

is called a weak (topological) equivalence between $V$ and $\tilde{V}$, if $h:\left(\mathbb{R}^{k}, 0\right) \rightarrow\left(\mathbb{R}^{k}, 0\right)$ is a germ of a homeomorphism of the parameter spaces, and each $H_{\alpha}$ is an orbital topological equivalence between $v_{\alpha}$ and $\tilde{v}_{h(\alpha)}$.

A weak topological equivalence $H$ is called

(strong) topological equivalence, if $H$ continuously depends on $(\alpha, x)$;

moderat $\mathrm{e}^{3}$ topological equivalence, if $H$ is continuous at every point $(0, x)$, where $x$ is either a singular point of $v_{0}$, or belongs to the union of all periodic orbits and separatrices of $v_{0}$, and $H^{-1}$ is continuous at every point of a similar set for $\tilde{V}$;

weak topological equivalence with $S e p$-tracing, if $H$ satisfies the following property. Let $S_{\alpha}, \alpha \in\left(\mathbb{R}^{k}, 0\right)$ be a continuous family of saddle points of $v_{\alpha}$, and $\left(\gamma_{\alpha}, S_{\alpha}\right)$ be a continuous family of local separatrices of these saddles. Then $H_{\alpha}\left(S_{\alpha}\right)$ and $H_{\alpha}\left(\left(\gamma_{\alpha}, S_{\alpha}\right)\right)$ are continuous families of saddle points and their local separatrices of $\tilde{v}_{h(\alpha)}$.

Pros and cons of these and some other equivalence relations are discussed in [12]. In this paper we will mostly use the last one.

\subsection{Main Theorems}

\subsubsection{Invariant functions and numerical invariants}

Let $\mathbf{M} \subset$ Vect be a Banach submanifold, $\operatorname{codim} \mathbf{M}<\infty$; let $k$ be a natural number, $k \geq \operatorname{codim} \mathbf{M}$. Denote by $\mathbf{M}^{\pitchfork, k} \subset \mathcal{V}_{k}$ the set of local families $V$ such that $v_{0} \in \mathbf{M}$ and $V$ is transverse to $\mathbf{M}$ at $v_{0}$. All numerical invariants of local families constructed in 16 , 18. follow the same pattern: they have the form $V \mapsto \varphi\left(v_{0}\right)$, where $\varphi: \mathbf{M} \rightarrow \mathbb{R}$ is an invariant function in the following sense.

Definition 4 (cf. [16, Definition 16]). Let $\sim$ be one of the equivalences on $\mathcal{V}_{k}$ defined above. A function $\varphi: \mathbf{M} \rightarrow \mathbb{R}$ defined on a Banach submanifold of Vect is called invariant with respect to $\sim$, if for any two $\sim$-equivalent local families $V, \tilde{V} \in \mathbf{M}^{\pitchfork, \text { codim } \mathbf{M}}$ we have $\varphi\left(v_{0}\right)=\varphi\left(\tilde{v}_{0}\right)$.

A function $\varphi: \mathbf{M} \rightarrow \mathbb{R}$ is called robustly invariant with respect to $\sim$, if the same equality holds for any two $\sim$-equivalent families $V, \tilde{V} \in \mathbf{M}^{\dagger, k}, k \geq \operatorname{codim} \mathbf{M}$.

\footnotetext{
${ }^{3}$ This definition works well only for families such that none of the vector fields have non-hyperbolic singular points. For a more general version, see 11 .
} 
By definition, if $\varphi$ is an invariant function with respect to $\sim$, then $V \mapsto \varphi\left(v_{0}\right)$ is a numerical invariant of classification of local families $V \in \mathbf{M}^{\pitchfork, c o d i m} \mathbf{M}$ with respect to $\sim$.

In order to transfer this invariant to an open set in the space of (non-local in parameter) families of vector fields $V: \mathcal{B} \rightarrow$ Vect, we require $\mathbf{M}$ to be topologically distinguished in the following sense.

Definition 5 (cf. [16, Definition 16]). We say that a Banach submanifold $\mathbf{M} \subset$ Vect is topologically distinguished in its neighbourhood $\mathcal{U} \supset \mathbf{M}$, if two vector fields $v \in \mathbf{M}$ and $w \in \mathcal{U} \backslash \mathbf{M}$ cannot be orbitally topologically equivalent.

In Sec. 4 we shall prove the following theorem.

Theorem 1. There exist a submanifold $\mathbf{M} \subset$ Vect and a smooth function $\varphi: \mathbf{M} \rightarrow \mathbb{R}$ such that

- $\mathbf{M}$ is topologically distinguished in its sufficiently small neighbourhood;

- $\varphi$ is robustly invariant with respect to the weak topological equivalence with Septracing;

- the image of $\varphi$ is the set of all positive numbers;

- for all $v \in \mathbf{M}, d \varphi(v) \neq 0$.

More precisely, one example of a pair $(\mathbf{M}, \varphi)$ with these properties is the submanifold $\mathbf{T}$ and the function $\nu$ from [16, Sec. 2.1.3]. We provide two new examples of pairs $(\mathbf{M}, \varphi)$ satisfying the conclusions of this theorem.

As explained above, the second conclusion of Theorem 1 implies that $V \mapsto \varphi\left(v_{0}\right)$ is an invariant of classification of families $V \in \mathbf{M}^{\text {巾, }}, k \geq 3$, up to the weak equivalence with Sep-tracing. The last two conclusions show that this invariant is non-degenerate; in particular, all families $V \in \mathbf{M}^{\dagger, k}$ are structurally unstable in $\mathbf{M}^{\pitchfork, k}$.

In Theorem 1 we prove that $\varphi$ is not just an invariant function, but a robustly invariant function for the sake of future applications, see Sec. 5 .

\subsubsection{Local and non-local families}

Let us explain how the first conclusion of Theorem 1 enables us to transfer this statement to an open set in the space of non-local families. We shall need a definition of the weak equivalence for non-local families, cf. Definition 3.

Definition 6. Two non-local families $V: \mathcal{B} \rightarrow$ Vect, $\tilde{V}: \tilde{\mathcal{B}} \rightarrow$ Vect, $\mathcal{B}, \tilde{\mathcal{B}} \subset \mathbb{R}^{k}$, are said to be weakly topologically equivalent, if there exists a homeomorphism $h: \mathcal{B} \rightarrow \tilde{\mathcal{B}}$ and a family of orientation-preserving homeomorphisms $H_{\alpha}: S^{2} \rightarrow S^{2}, \alpha \in \mathcal{B}$, such that for each $\alpha$, the germ of $H:(\alpha, x) \mapsto\left(h(\alpha), H_{\alpha}(x)\right)$ at this value of $\alpha$ is a weak equivalence between the local families $(V, \alpha)$ and $(\tilde{V}, h(\alpha))$.

Other equivalences can be similarly transferred to the class of non-local families.

Let $\mathbf{M} \subset$ Vect be a Banach submanifold that is topologically distinguished in its neighbourhood $\mathcal{U}$. Suppose that a function $\varphi: \mathbf{M} \rightarrow \mathbb{R}$ is invariant with respect to $\sim$. Consider the space $\mathbf{M}_{\text {nonloc. }}^{\dagger}$ of $k$-parameter non-local families $V=\left\{v_{\alpha}\right\}_{\alpha \in \mathcal{B}}, k=$ $\operatorname{codim} \mathbf{M}$, with the following properties. 


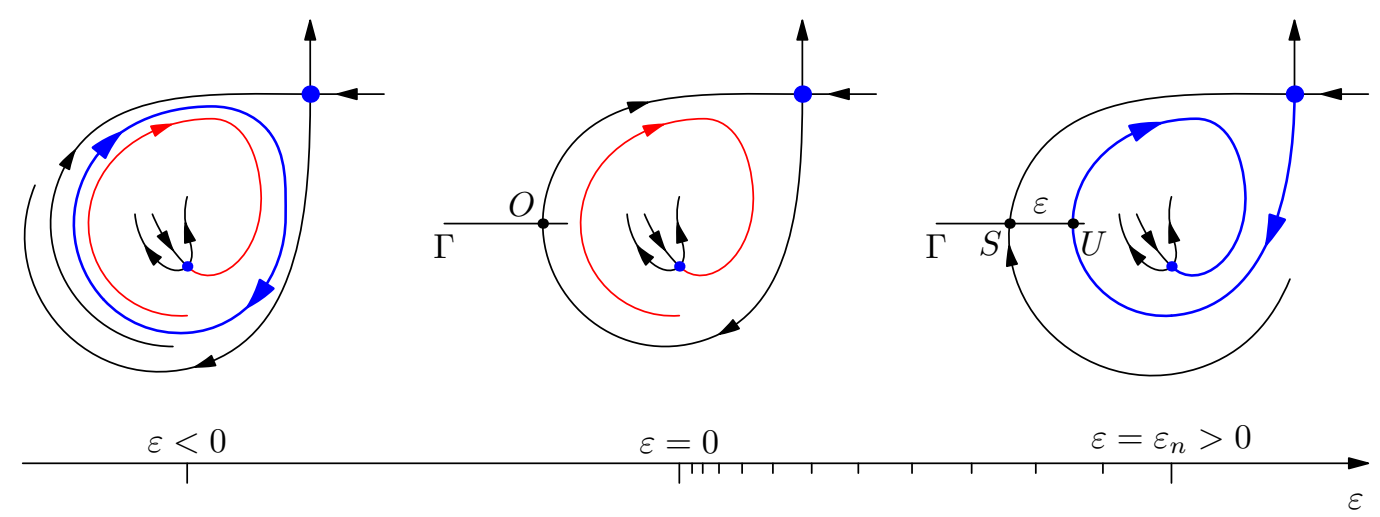

Figure 3: Saddle loop bifurcation

- $v_{\alpha} \in \mathcal{U}$ for all $\alpha \in \mathcal{B}$;

- $V$ meets $\mathbf{M}$ at a single vector field $v$;

- $V$ is transverse to $\mathbf{M}$ at $v$.

Since $\mathbf{M}$ is topologically distinguished, the map $\mathbf{M}_{\text {nonloc. }}^{\dagger} \rightarrow \mathbf{M}^{\dagger, k}$ given by $V \mapsto$ $(V, V \cap \mathbf{M})$ sends $\sim$-equivalent non-local families to $\sim$-equivalent local families. Therefore, the formula $V \mapsto \varphi(V \cap \mathbf{M})$ defines a numerical invariant of classification of non-local families $V \in \mathbf{M}_{\text {nonloc. }}^{\dagger}$ up to the $\sim$-equivalence.

Applying these arguments to Theorem 1, one can prove the following theorem.

Theorem 2. There exists an open subset in the space of non-local 3-parameter families of vector fields on the sphere such that the classification of these families up to the weak topological equivalence with Sep-tracing has a numerical invariant. In particular, families from this open set are not structurally stable.

\section{Saddle loop bifurcation}

\subsection{Statement of the theorem and corollaries}

Recall that for a hyperbolic saddle point $L$ of a vector field $v \in$ Vect, its characteristic number is the absolute value of the ratio of the eigenvalues of the linearization of $v$ at $L$, the negative one is in the numerator.

Consider a vector field $v_{0} \in$ Vect with a hyperbolic saddle $L$. Suppose that

- the characteristic number $\lambda$ of $L$ is greater than one, $\lambda>1$;

- an unstable separatrix $l^{u}$ of $L$ forms a separatrix loop $l$ with a stable separatrix $l^{s}$ of the same saddle;

- an unstable separatrix $\gamma$ of another hyperbolic saddle $I$ winds onto $l$.

Given a vector field $v \in$ (Vect, $v_{0}$ ), let $L(v)$ and $I(v)$ be the hyperbolic saddles of $v$ close to $L$ and $I$; let $l^{u}(v), l^{s}(v)$, and $\gamma(v)$ be separatrices of these saddles such that the germs $\left(l^{u}(v), L(v)\right),\left(l^{s}(v), L(v)\right)$, and $(\gamma(v), I(v))$ are close to the germs $\left(l^{u}, L\right),\left(l^{s}, L\right)$, and 
$(\gamma, I)$, respectively. Fix a cross-section $(\Gamma, O)$ to $l$ and a coordinate $x:(\Gamma, O) \rightarrow(\mathbb{R}, 0)$ so that $x$ is positive on $\Gamma \cap \gamma$; let $S(v)$ and $U(v)$ be the first intersection points of $l^{s}(v)$ and $l^{u}(v)$ with this cross-section, counting from $L$. The difference

$$
\varepsilon(v)=x(S(v))-x(U(v))
$$

is called the separatrix splitting parameter for the separatrix loop $l$, see Fig. 3 .

Denote by $\left(\mathbf{S L}_{l}, v_{0}\right) \subset$ (Vect, $\left.v_{0}\right)$ the germ of the codimension-one Banach submanifold of Vect given by $\varepsilon(v)=0$. Geometrically, this equation means that $l^{u}(v)$ coalesces with $l^{s}(v)$, so the separatrix loop $l$ survives.

Consider an unfolding $V=\left\{v_{\alpha}\right\}_{\alpha \in\left(\mathbb{R}^{k}, 0\right)}, k \geq 1$, of $v_{0}$ transverse to $\left(\mathbf{S L}_{l}, v_{0}\right)$. Let us reparametrize this family so that $\alpha=(\varepsilon, \beta)$. The objects introduced above $\left(L, I, l^{s}, l^{u}\right.$, $S, U, \varepsilon)$ can be considered as functions of $\alpha: \varepsilon(\alpha):=\varepsilon\left(v_{\alpha}\right)$ etc.

Consider another vector field $\tilde{v}_{0}$ of the same type, its saddle loop $\tilde{l}$, and an unfolding $\tilde{V}$ of $\tilde{v}_{0}$ transverse to $\mathbf{S L}_{\tilde{l}}$. Denote by $\tilde{L}$ etc. the objects that play the same role for $\tilde{V}$ as the corresponding objects for $V$.

The following theorem reinterprets results of [16] as a reusable statement incapsulating usage of sparkling separatrix connections.

Theorem 3. In the settings introduced above, suppose that $H:(\alpha, x) \mapsto\left(h(\alpha), H_{\alpha}(x)\right)$ is a weak equivalence between $V$ and $\tilde{V}$ such that $H_{\alpha}$ sends $L(\alpha), l^{s}(\alpha), l^{u}(\alpha), I(\alpha)$, and $\gamma(\alpha)$ to $\tilde{L}(h(\alpha)), \tilde{l}^{s}(h(\alpha)), \tilde{l}^{u}(h(\alpha)), \tilde{I}(h(\alpha))$, and $\tilde{\gamma}(h(\alpha))$, respectively. Then for $\varepsilon>0$, $(\tilde{\varepsilon}, \tilde{\beta})=h(\varepsilon, \beta)$ the difference

$$
\frac{\ln (-\ln \tilde{\varepsilon})}{\ln \tilde{\lambda}(0, \tilde{\beta})}-\frac{\ln (-\ln \varepsilon)}{\ln \lambda(0, \beta)}
$$

is uniformly bounded in $\{(\varepsilon, \beta) \mid 0<\varepsilon<C,\|\beta\|<C\}$ for some $C>0$, and

$$
\lim _{\substack{\varepsilon \rightarrow 0+\\ \beta \rightarrow 0}} \frac{\ln (-\ln \tilde{\varepsilon})}{\ln (-\ln \varepsilon)}=\frac{\ln \tilde{\lambda}(0)}{\ln \lambda(0)} .
$$

Geometrically, $\frac{\ln (-\ln \varepsilon)}{\ln \lambda(0, \beta)}$ is the number of turns $\gamma$ makes around $l$ before coming to the interval $[S(\tilde{v}), U(\tilde{v})) \subset \Gamma$.

Before we prove this theorem, let us use it to prove two corollaries.

Corollary 1 (cf. [9]). In the settings of Theorem 3, suppose that both $h$ and $h^{-1}$ are Hölder continuous. Then $\lambda(0)=\tilde{\lambda}(0)$.

Proof. Choose $C$ and $\kappa$ such that both $h$ and $h^{-1}$ are Hölder continuous with Hölder coefficient $C$ and Hölder exponent $\kappa$. Then for $(\tilde{\varepsilon}, \tilde{\beta})=h(\varepsilon, \beta)$ we have

$$
|\tilde{\varepsilon}| \leq\|(\tilde{\varepsilon}, \tilde{\beta})-h(0, \beta)\| \leq C\|(\varepsilon, \beta)-(0, \beta)\|^{\kappa}=C|\varepsilon|^{\kappa},
$$

and similarly $|\varepsilon| \leq C|\tilde{\varepsilon}|^{\kappa}$. These inequalities imply that the left hand side of (2) equals one, thus $\lambda(0,0)=\tilde{\lambda}(0,0)$. 
Corollary 2. Let $\mathbf{S L} \subset$ Vect be the Banach manifold of vector fields $v \in$ Vect of the type described above with no other degeneracies. Then $\lambda: \mathbf{S L} \rightarrow \mathbb{R}_{+}$is a robustly invariant function of classification of unfoldings $V \in \mathbf{S L}^{\pitchfork, k}, k \geq 1$, up to the weak equivalence with Sep-tracing and additional restriction "both $h$ and $h^{-1}$ are Hölder continuous".

This corollary immediately follows from Corollary 1.

Remark 1 . Theorem 3 and the corollaries can be easily generalized to the case of vector fields with a monodromic hyperbolic polycycle $\Pi$, with a few modifications:

- $l$ is a separatrix connection of $\Pi$, not necessarily a separatrix loop;

- $\varepsilon$ and $\tilde{\varepsilon}$ are separatrix splitting parameters corresponding to $l$ and $\tilde{l}=H_{0}(l)$;

- (2) holds on the subspace given by the condition "all other separatrix connections of $\Pi$ survive";

- $H$ is a moderate equivalence between $V$ and $\tilde{V}$; otherwise $h$ may a priori send the subspace described above of $V$ to another subspace of the parameter space of $\tilde{V}$.

In the rest of this section we prove Theorem 3.

\subsection{Sparkling separatrix connections}

Consider a vector field $v \in$ (Vect, $\left.v_{0}\right)$. If $\varepsilon(v)=0$, then the separatrix loop $l$ survives. If $\varepsilon(v)<0$, then an attracting hyperbolic limit cycle appears near $l$, and this limit cycle separates $l^{u, s}(v)$ from $\gamma(v)$.

In the last case $\varepsilon(v)>0$, the separatrix $\gamma(v)$ makes several turns around $l$, then comes to the interval $[S(v), U(v))$. The coordinate change by an appropriate Dehn twist changes the number of turns $\gamma(v)$ makes around $l$ by one, so this number $N(v)$ is not a well-defined function of a vector field $v \in\left(\right.$ Vect, $\left.v_{0}\right)$. However, we can define $N(v)$ up to an additive constant, cf. [16, Definition 13, 18, Remark 2]. Namely, we fix an intersection point $P \in \gamma \cap \Gamma$, then choose a continuous family of points $P(v) \in \gamma(v) \cap \Gamma, P\left(v_{0}\right)=P$, and let $N(v)$ be the cardinality of $\gamma(v) \cap(U(v), P(v)] \subset \gamma(v) \cap \Gamma$. Clearly, a different choice of $\Gamma$ and/or $P$ leads to a function of the form $N(v)+$ const, hence $N$ is defined up to an additive constant.

From now on, we fix $\Gamma$ and $P$, hence the function $N$. This function has step discontinuities at vector fields $v$ such that $S(v) \in \gamma(v)$. This means that $\gamma(v)$ makes $N(v)$ turns around $l$, then coalesces with $l^{s}(v)$.

Given a local family $V$ as in Theorem 3 , denote by $\mathcal{C}$ the set of values of $\alpha$ such that $v_{\alpha}$ has a separatrix connection described in the previous paragraph. This set is a union of connected components $\mathcal{C}_{n}$ enumerated by $N(\alpha):=N(v(\alpha))$.

Due to [16, Lemma 4], in some small neighborhood of the origin, each $\mathcal{C}_{n}$ is the graph of a function $\varepsilon=\varepsilon_{n}(\beta)$. In other words, for each $\beta$ from a small neighborhood of the origin and $n$ large enough, there exists a unique $\varepsilon=\varepsilon_{n}(\beta)$ such that $v_{\alpha}=v_{\varepsilon, \beta}$ has a separatrix connection between $\gamma(\alpha)$ and $l^{s}(\alpha)$ with $n$ turns. Moreover, these functions form a decreasing functional sequence such that

$$
\ln \left(-\ln \varepsilon_{n}(\beta)\right)=n \ln \lambda(0, \beta)+O(1),
$$


see also [18, Lemma 6] for a more precise estimate. Here $O(1)$ term in the right hand side of (3) is uniformly bounded in some neighborhood $\{(\varepsilon, \beta) \mid 0<\varepsilon<C,\|\beta\|<C\}$.

Note that $N(\alpha), \alpha=(\varepsilon, \beta)$, can be equivalently defined by the inequalities

$$
\varepsilon_{N(\alpha)+1}(\beta) \leq \varepsilon<\varepsilon_{N(\alpha)}(\beta) .
$$

Then (3) implies that

$$
N(\varepsilon, \beta)=\frac{\ln (-\ln \varepsilon)}{\ln \lambda(0, \beta)}+O(1) \quad \text { as } \varepsilon \rightarrow 0+\quad \text { uniformly in } \beta .
$$

In the next section we shall use this fact to prove Theorem 3.

\subsection{Comparing two families}

Consider two equivalent families $V$ and $\tilde{V}$ as in Theorem 3. Let $\mathcal{C}$ be the set defined above, and $\tilde{\mathcal{C}}$ be the similar set for $\tilde{V}$. Since $H_{\alpha}$ sends $\gamma(v)$ and $l^{s}(v)$ to $\tilde{\gamma}(\tilde{v})$ and $\tilde{l}^{s}(\tilde{v})$, we have $h(\mathcal{C})=\tilde{\mathcal{C}}$. Since $h$ is a homeomorphism, it sends each connected component $\mathcal{C}_{n}$ to a connected component $\tilde{\mathcal{C}}_{n^{\prime}}$, possibly with a different index. However, it preserves the relative order of these connected components, hence there exists a constant $a \in \mathbb{Z}$ such that for $n$ large enough we have $h\left(\mathcal{C}_{n}\right)=\tilde{\mathcal{C}}_{n+a}$. Recall that the function $N$ defined above is defined up to an additive constant, so we may and will assume that $\tilde{N}(h(\alpha))=N(\alpha)$.

Now take $(\tilde{\varepsilon}, \tilde{\beta})=h(\varepsilon, \beta)$, and substitute asymptotic estimates (4) both for $N(\varepsilon, \beta)$ and $\tilde{N}(\tilde{\varepsilon}, \tilde{\beta})$ in the formula $\tilde{N}(\tilde{\varepsilon}, \tilde{\beta})=N(\varepsilon, \beta)$. This immediately implies that the difference (1) is uniformly bounded in some neighborhood $\{(\varepsilon, \beta) \mid 0<\varepsilon<C,\|\beta\|<C\}$.

In order to prove (2), it suffices to apply the estimate from the previous paragraph, and use the fact that $\ln (-\ln \varepsilon) \rightarrow \infty$ as $\varepsilon \rightarrow 0$.

\section{Vector fields with "ears" or "glasses"}

In this section we prove Theorem 1. In Sec. 4.1, we describe $\mathbf{M}$ and $\varphi$. In Sec. 4.2 we consider two equivalent families $V, V \in \mathbf{M}^{\dagger, k}, k \geq 3$, and use Theorem 3 to deduce an asymptotic relation on the components of $\alpha$ and $h(\alpha)$. Finally, in Sec. 4.3 we describe a special "synchronizing" subfamily; restriction of the relation from Sec. 4.2 to this subfamily implies $\varphi\left(v_{0}\right)=\varphi\left(\tilde{v}_{0}\right)$.

\subsection{Special classes of degenerate vector fields}

The manifold $\mathbf{M}$ consists of two very similar disjoint components $\mathbf{E}$ and $\mathbf{G}$ (from "ears" and "glasses"), $\mathbf{M}=\mathbf{E} \sqcup \mathbf{G}$, see Fig. 2. We shall describe these components simultaneously.

\subsubsection{Separatrix graphs "ears" and "glasses"}

Consider a vector field $v$. Suppose that it has two hyperbolic saddles $L$ and $R$ (from "left" and "right"), and the following separatrix connections: 
- saddles $L$ and $R$ have separatrix loops $l$ and $r$, respectively;

- the "unused" unstable separatrix of $L$ coalesces with the "unused" stable separatrix of $R$, forming a separatrix connection $b$ (from "bridge").

The loops $l$ and $r$ split the sphere into two discs and one annulus. Mark one of the points in the annulus as "infinity", $\infty \notin b$, and make a stereographic projection $S^{2} \backslash\{\infty\} \rightarrow \mathbb{R}^{2}$. Then we can talk about various points being "inside" or "outside" some curves.

In particular, orientation of the loops $l, r$ ("clockwise" or "counter-clockwise") is welldefined. If a vector field has saddles and separatrix connections described above, we say that it has a separatrix graph "ears" or "glasses" depending on the orientation of $l$ and $r$, namely

for "glasses", the loops $l$ and $r$ are oriented in the same way;

for "ears", the loops $l$ and $r$ are oriented in the opposite ways.

\subsubsection{Winding separatrices}

Assume that the characteristic numbers $\lambda$ and $\rho$ of $L$ and $R$ satisfy the inequalities

$$
\lambda>1, \quad \rho<1 .
$$

These inequalities imply that the loop $l$ attracts from the inside, while the loop $r$ repels from the inside, see [16, Remark 12]. Next, assume that there is a hyperbolic saddle point $I_{L}$ inside $l$, and one of the unstable separatrices $\gamma_{L}$ of $I_{L}$ winds onto $l$. Formally, the $\omega$-limit set of $\gamma_{L}$ is $l \cup\{L\}$. Similarly, there is a hyperbolic saddle $I_{R}$ inside $r$, and one of its stable separatrices $\gamma_{R}$ winds onto $r$ in the reverse time.

This completes the description of the "interesting" part of the phase portrait. For technical reasons, we also require that the rest of the phase portrait is "not interesting", i.e. structurally stable.

Definition 7. We say that a vector field $v$ belongs to $\mathbf{E}$ (resp. G), if

1. it has a separatrix graph "ears" (resp. "glasses");

2. the characteristic numbers $\lambda$ and $\rho$ of $L$ and $R$ satisfy the inequalities (5);

3. it has hyperbolic saddle points $I_{L}, I_{R}$, and their separatrices $\gamma_{L}$ and $\gamma_{R}$ winding onto $l$ and $r$ as described above;

4. the unused stable separatrix of $L$ tends to a hyperbolic source or a hyperbolic repelling cycle in the reverse time, and the unused unstable separatrix of $R$ tends to a hyperbolic sink or a hyperbolic attracting cycle in the forward time;

5. the restriction of $v$ to the complement of a small neighborhood of $l \cup b \cup r$ is structurally stable.

For a vector field $v \in \mathbf{E} \cup \mathbf{G}$, we put

$$
\varphi(v)=-\frac{\ln \rho(v)}{\ln \lambda(v)} .
$$


Recall that $\mathbf{M}=\mathbf{E} \sqcup \mathbf{G}$. It is easy to see that $\mathbf{M}$ is an embedded Banach submanifold of Vect of codimension 3, i.e. near each vector field $v \in \mathbf{M}$ there exists a germ of a smooth map $\psi$ : (Vect, $v) \rightarrow\left(\mathbb{R}^{3}, 0\right)$ of full rank such that $(\mathbf{M}, v)=\left(\psi^{-1}(0), v\right)$. Indeed, it suffices to take $\psi=(\varepsilon, \sigma, \delta)$, where $\varepsilon, \sigma$, and $\delta$ are the separatrix splitting parameters for $l, b, r$, respectively, defined as in Sec. 3.1. Note that a similar statement is false at some points of the closure of $\mathbf{M}$ in Vect, e.g. those corresponding to vector fields with two "ears" graphs at different locations.

The following theorem is an explicit version of Theorem 1 .

Theorem 4. The function $\varphi: \mathbf{M} \rightarrow \mathbb{R}$ defined above satisfies all conclusions of Theorem 1.

Clearly, $\varphi(\mathbf{M})$ is the set of positive numbers, and $d \varphi$ does not vanish anywhere on $\mathbf{M}$. The last two requirements of Definition 7 guarantee that $\mathbf{M}$ is topologically distinguished in its sufficiently small neighborhood. So, it remains to show that $\varphi$ is robustly invariant with respect to the weak topological equivalence with $S e p$-tracing.

\subsubsection{Technical hypotheses}

We shall prove Theorem 4 in slightly more general settings. Namely, we want to isolate parts of the proof that use two technical hypotheses: the Sep-tracing property and structural stability of $v$ away from the separatrix graph "ears" or "glasses", see Definition 7.

In order to get rid of these two requirements, we consider vector fields with marked saddles $L, R, I_{L}, I_{R}$ and separatrices $\gamma_{L}, \gamma_{R}$, and require that $H_{\alpha}$ sends these objects for $v_{\alpha}$ to the corresponding objects for $\tilde{v}_{h(\alpha)}$.

Namely, consider a vector field $v$, its hyperbolic saddles $L, R, I_{L}, I_{R}$, and separatrices $l, b, r, \gamma_{L}, \gamma_{R}$ that satisfy the first four requirements of Definition 7. For a small perturbation of $v$, condition "separatrix connections $l, b, r$ survive" defines a germ of a Banach submanifold $\left(\mathbf{M}_{L, R}, v\right) \subset$ (Vect, $\left.v\right)$ of codimension 3, and (6) defines a germ of a smooth function $\varphi_{L, R}:\left(\mathbf{M}_{L, R}, v\right) \rightarrow\left(\mathbb{R}, \varphi_{L, R}(v)\right)$. In the case $v \in \mathbf{M}$, these germs concide with $(\mathbf{M}, v)$ and $\left.\varphi\right|_{(\mathbf{M}, v)}$, respectively.

Let $V=\left\{v_{\alpha}\right\}_{\alpha \in\left(\mathbb{R}^{k}, 0\right)} \in \mathbf{M}_{L, R}^{\dagger, k}, k \geq 3$, be an unfolding of $v=v_{0}$ transverse to $\mathbf{M}_{L, R}$. A vector field $v_{\alpha}$ has saddle points $L(\alpha), R(\alpha), I_{L}(\alpha), I_{R}(\alpha)$ close to the saddles $L, R$, $I_{L}, I_{R}$ of $v=v_{0}$, and separatrices $\gamma_{L}(\alpha), \gamma_{R}(\alpha)$ close to corresponding separatrices of $v$. Separatrix connections $l, b, r$ of $v_{0}$ are possibly destroyed, and each of them generates two continuous families of separatrices, one stable and one unstable. Denote by $l^{s}(\alpha)$, $l^{u}(\alpha), b^{s}(\alpha), b^{u}(\alpha), r^{s}(\alpha), r^{u}(\alpha)$ these families, where superscript denotes (un)stability of the separatrix.

Let $\tilde{V}$ be another family of vector field with marked saddles and separatrices of the same type. By letters with tilde above we denote objects for $\tilde{V}$ similar to those objects for $V$ denoted by the same letter without tilde.

The following theorem is a slightly generalized version of Theorem 4 that does not rely on the two technical assumptions mentioned above. 
Theorem 5. In the settings introduced above, suppose that $H:(\alpha, x) \mapsto\left(h(\alpha), H_{\alpha}(x)\right)$ is a weak topological equivalence between $V$ and $\tilde{V}$ such that $H_{\alpha}$ sends $L(\alpha), R(\alpha), l^{s, u}(\alpha)$, $b^{s, u}(\alpha), r^{s, u}(\alpha), \gamma_{L}(\alpha), \gamma_{R}(\alpha)$ to $\tilde{L}(h(\alpha)), \tilde{R}(h(\alpha))$ etc. Then $\varphi_{L, R}\left(v_{0}\right)=\varphi_{\tilde{L}, \tilde{R}}\left(\tilde{v}_{0}\right)$.

Let us show that this theorem implies Theorem 4. Consider two families $V, \tilde{V} \in \mathbf{M}^{\dagger, k}$, $k \geq 3$. Suppose that they are weakly topologically equivalent with $S e p$-tracing property. Fix all saddles and separatrices from Sec. 4.1.2 for $V$, and introduce families $L(\alpha)$ etc. as above. Due to the $S e p$-tracing property, the images of these families under $H_{\alpha}$ are families with similar properties for $\tilde{V}$. Thus we can apply Theorem 5 to these two families, and the assumptions $H_{\alpha}(L(\alpha))=\tilde{L}(h(\alpha))$ etc. will hold automatically.

Finally, due to the last assumption of Definition 7, $\tilde{v}_{0}$ has a unique separatrix graph "ears" or "glasses", hence $\tilde{L}$ and $\tilde{R}$ are the saddles from Definition 7 for $\tilde{v}_{0}$, thus conclusion of Theorem 5 matches the only non-trivial part of the conclusion of Theorem 4.

Remark 2. Theorem 5 compared to Theorem 4 replaces a pair of technical assumptions with another one, namely $H_{\alpha}(L(\alpha))=\tilde{L}(h(\alpha))$ etc. Another approach would be to impose more assumptions on the families $V, \tilde{V}$ while still working with the weak equivalence. E.g., one can "tag" all hyperbolic sinks and sources by surrounding each of them with some number of hyperbolic cycles. If the number of cycles is different for each "nest", then $H_{\alpha}$ has to send each nest of $v_{\alpha}$ to the corresponding nest of $\tilde{v}_{h(\alpha)}$, and all we have to do is to "identify" saddle points and their separatrices. In some cases, it is possible to identify them based on the $\alpha$-limit and $\omega$-limit sets of the separatrices; in other cases it might need more subtle topological arguments.

\subsection{Sparkling separatrix connections}

Consider two families $V, \tilde{V}$ with marked saddles and separatrices as described in Sec. 4.1.3. Let $H$ be a weak equivalence between $V$ and $\tilde{V}$ that satisfies the assumptions of Theorem 5.

In order to use the presence of the separatrices $\gamma_{L}, \gamma_{R}$, and sparkling separatrix connections that appear when we destroy the separatrix loops, we apply Theorem 3 to $V, \tilde{V}$, and $H$ twice. First, we apply this theorem to the separatrix loops $l$ and $\tilde{l}$, and get

$$
\frac{\ln (-\ln \tilde{\varepsilon})}{\ln (-\ln \varepsilon)} \rightarrow \frac{\ln \tilde{\lambda}(0)}{\ln \lambda(0)}
$$

as $\varepsilon \rightarrow 0+,(\sigma, \delta, \eta) \rightarrow 0,(\tilde{\varepsilon}, \tilde{\sigma}, \tilde{\delta}, \tilde{\eta})=h(\varepsilon, \sigma, \delta, \eta)$.

Second, we want to apply the same theorem to $r$ and $\tilde{r}$. Since $\rho<1$ and $\gamma_{R}$ winds onto $r$ in the reverse time, this theorem does not apply literally, so we first reverse time by replacing $v_{\alpha}$ with $-v_{\alpha}$, and get

$$
\frac{\ln (-\ln \tilde{\delta})}{\ln (-\ln \delta)} \rightarrow \frac{\ln \tilde{\rho}(0)^{-1}}{\ln \rho(0)^{-1}}
$$

as $\delta \rightarrow 0+,(\varepsilon, \sigma, \eta) \rightarrow 0,(\tilde{\varepsilon}, \tilde{\sigma}, \tilde{\delta}, \tilde{\eta})=h(\varepsilon, \sigma, \delta, \eta)$. 

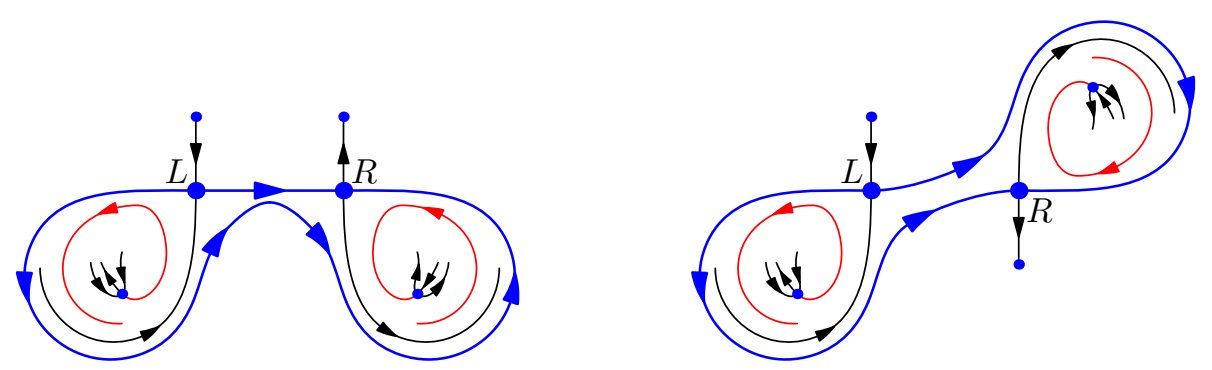

Figure 4: Vector fields $v_{\alpha}, \alpha \in \mathcal{E}$, for "glasses" and "ears"

Dividing (7) by (8), we obtain

$$
\frac{\ln (-\ln \tilde{\varepsilon})}{\ln (-\ln \tilde{\delta})} \div \frac{\ln (-\ln \varepsilon)}{\ln (-\ln \delta)} \rightarrow \frac{\ln \rho(0)^{-1}}{\ln \lambda(0)} \div \frac{\ln \tilde{\rho}(0)^{-1}}{\ln \tilde{\lambda}(0)}=\frac{\varphi_{L, R}\left(v_{0}\right)}{\varphi_{\tilde{L}, \tilde{R}}\left(\tilde{v}_{0}\right)}
$$

as $\varepsilon \rightarrow 0+, \delta \rightarrow 0+, \sigma \rightarrow 0, \eta \rightarrow 0,(\tilde{\varepsilon}, \tilde{\sigma}, \tilde{\delta}, \tilde{\eta})=h(\varepsilon, \sigma, \delta, \eta)$. In order to complete the proof of Theorem 5 it suffices to find a synchronizing subfamily $\mathcal{E} \subset\left\{\alpha \in\left(\mathbb{R}^{k}, 0\right) \mid \varepsilon>0, \delta>0\right\}$ such that

$$
\lim _{\substack{\alpha \rightarrow 0 \\ \alpha \in \mathcal{E}}} \frac{\ln (-\ln \varepsilon)}{\ln (-\ln \delta)}=\lim _{\substack{\alpha \rightarrow 0 \\ \alpha \in \mathcal{E}}} \frac{\ln (-\ln \tilde{\varepsilon})}{\ln (-\ln \tilde{\delta})}=1,
$$

where as usual $(\tilde{\varepsilon}, \tilde{\sigma}, \tilde{\delta}, \tilde{\eta})=h(\varepsilon, \sigma, \delta, \eta)$.

\subsection{Synchronizing subfamily}

The synchronizing subfamily $\mathcal{E} \subset\left(\mathbb{R}^{k}, 0\right)$ is defined by the following condition: $\alpha \in \mathcal{E}$ if and only if $v_{\alpha}$ has two different separatrix connections joining $L(\alpha)$ to $R(\alpha)$. Due to Assumption 4 of Definition 7, no orbit can start near $R$ and then come to a neighbourhood of $L$. Thus there is only one possibility for geometry of these two connections, see Fig. 4. In particular, $\alpha \in \mathcal{E}$ implies $\varepsilon>0$ and $\delta>0$.

Fig. 5 shows parts of the bifurcation diagram of "glasses" that are relevant to our proof, either directly, or through the proof of Theorem 3. In Fig. 5, we intentionally omit the curves corresponding to the degeneracies not used in our proof.

Lemma 1. For the subfamily $\mathcal{E}$ defined above, we have

$$
\frac{\ln (-\ln \varepsilon)}{\ln (-\ln \delta)} \rightrightarrows 1 \quad \text { as } \varepsilon, \delta \rightarrow 0+, \quad \alpha \in \mathcal{E} \quad \text { uniformly in } \eta .
$$

We shall prove this lemma separately for $\mathbf{M}=\mathbf{G}$ and for $\mathbf{M}=\mathbf{E}$.

Proof of Lemma 1 in the "glasses" case. It is easy to see that one of these connections is a perturbed bridge $b$, and the other is close to the union $l \cup b \cup r$. The former connection exists if and only if $\sigma=0$, and the latter connection exists if and only if

$$
S_{r}(\alpha)=\Delta\left(U_{l}(\alpha)\right)
$$




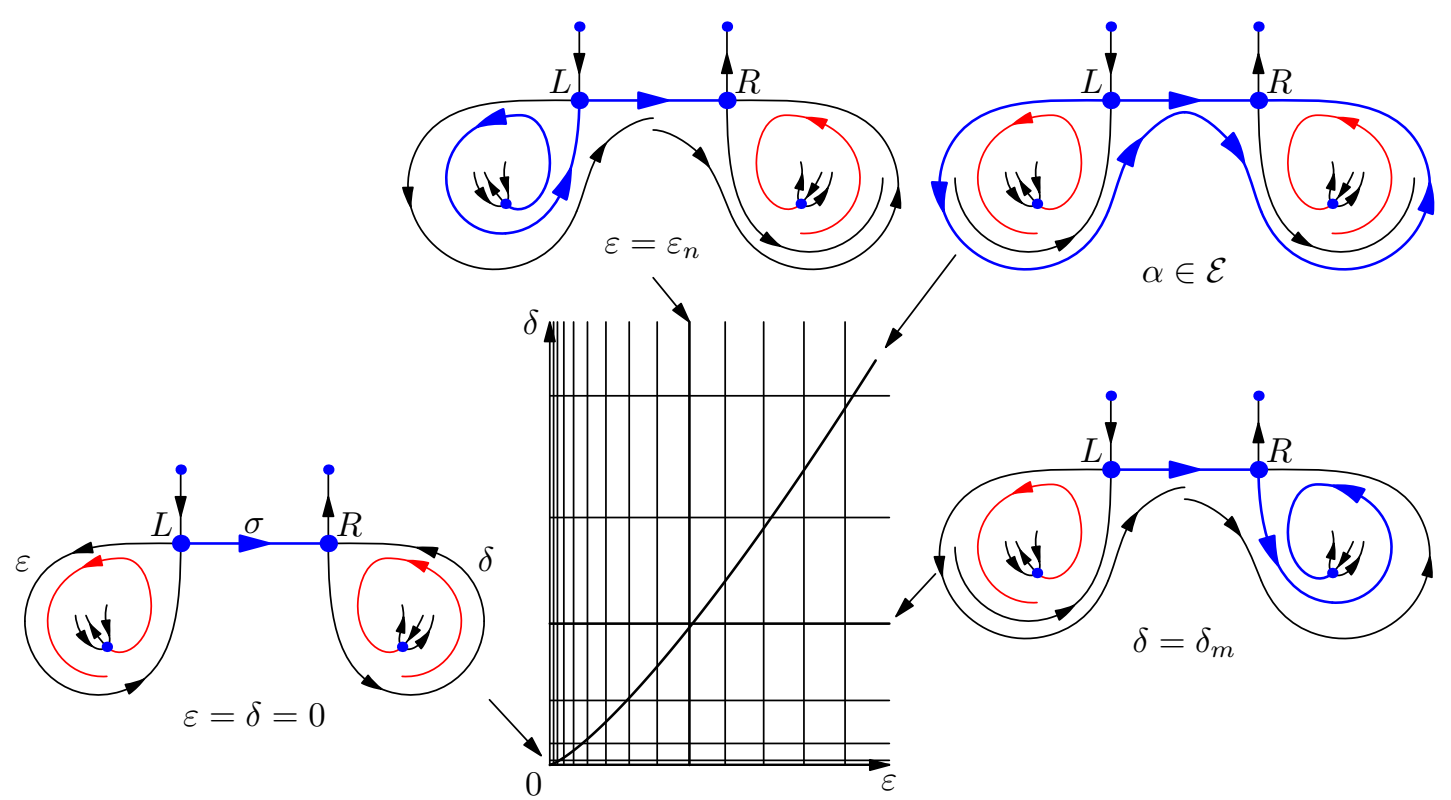

Figure 5: Parts of the bifurcation diagram of "glasses" in the plane $\sigma=0$ that are relevant to our proof

where $\Delta$ is the correspondence map from the "outer" half of $\Gamma_{l}$ to the "outer" half of $\Gamma_{r}$. By definition, $U_{l}(\alpha)$ is at the distance $\varepsilon$ from $S_{l}(\alpha)$, and $S_{r}(\alpha)$ is at the distance $\delta$ from $U_{r}(\alpha)$, hence in natural coordinates on $\Gamma_{l}, \Gamma_{r}$, we have $\delta=\Delta(\varepsilon)$.

Recall that the correspondence map of a hyperbolic saddle with characteristic number $\mu$ is close to $x \mapsto C x^{\mu}$, see e.g. [16, Lemma 5, 10, Lemma 1], and for a chain of maps one should multiply the exponents. Therefore, $\delta=\Delta(\varepsilon)$ implies that

$$
\ln \delta=\lambda(\alpha) \rho(\alpha) \ln \varepsilon+O(1)
$$

as $\varepsilon \rightarrow 0, \delta \rightarrow 0, \alpha \in \mathcal{E}$. Taking logarithms of both sides, we get (9).

Proof of Lemma 1 in the "ears" case. In this case, one of the separatrix connections is close to $l \cup b$, and the other is close to $b \cup r$. These connections exist if

$$
S_{b}(\alpha)=\Delta_{l}\left(U_{l}(\alpha)\right), \quad S_{r}(\alpha)=\Delta_{r}\left(U_{b}(\alpha)\right),
$$

where $\Delta_{l}$ is the correspondence map from the "outer" half of $\Gamma_{l}$ to $\Gamma_{b}$, and $\Delta_{r}$ is the correspondence map from one half of $\Gamma_{b}$ to the "outer" half of $\Gamma_{r}$.

Similarly to the previous case, these equalities imply

$$
\ln \sigma=\lambda(\alpha) \ln \varepsilon+O(1), \quad \ln \delta=\rho(\alpha) \ln \sigma+O(1) .
$$

Hence we have $(10)$, then complete the proof as in the previous case. 
Finally, recall that $H_{\alpha}$ sends separatrices of $L(\alpha)$ and $R(\alpha)$ to the corresponding separatrices of $\tilde{L}(h(\alpha))$ and $\tilde{R}(h(\alpha))$, hence $h(\mathcal{E})=\tilde{\mathcal{E}}$. Thus

$$
\lim _{\substack{\alpha \rightarrow 0 \\ \alpha \in \mathcal{E}}} \frac{\ln (-\ln \tilde{\varepsilon})}{\ln (-\ln \tilde{\delta})}=\lim _{\substack{\tilde{\alpha} \rightarrow 0 \\ \tilde{\alpha} \in \mathcal{E}}} \frac{\ln (-\ln \tilde{\varepsilon})}{\ln (-\ln \tilde{\delta})}=1 .
$$

This and the arguments at the end of Sec. 4.2 complete the proof of Theorem 5 , hence of Theorem 4 and of Theorem 1 .

\section{Future plans}

The statement and the main idea of the proof of Theorem 4 resemble the study of "tears of the heart" polycycle in 16. However, note that for "ears" and "glasses", the separatrix graph is not a polycycle: we can move only from the "left" half of the picture to the "right" half, not the other way around, which makes the bifurcation diagram simpler.

The first two authors plan to use this difference to prove the following facts.

1. There exists a submanifold $\mathbf{M} \subset$ Vect of codimension 3 such that the classification of local families $V \in \mathbf{M}^{\pitchfork, 3}$ has infinitely many invariants.

2. For every $d>0$ there exists a submanifold $\mathbf{M} \subset$ Vect of codimension $d+2$ that admits $d$ independent robustly invariant functions.

3. For every $d>0$ there exists a submanifold $\mathbf{M} \subset$ Vect of codimension 4 that admits $d$ independent robustly invariant functions.

4. There exists a submanifold $\mathbf{M} \subset$ Vect of finite codimension that admits infinitely many robustly invariant functions.

Both the second and the third statements imply that there exists an open set in the space of 5-parameter non-local families of vector fields on the sphere such that classification of these families has a functional invariant. This reduces the codimension in [16. Theorem 2] by one, and for $d>1$ we get an even better reduction in codimension compared to [16, Theorem 3].

Another example of locally generic 5-parameter families with functional invariants was recently found by Dukov [7, 8]. His example is a modification of the original "tears of the heart" polycycle.

The last statement from the list implies that vector fields $v \in \mathbf{M}$ have no finite parameter versal deformations, not even with the number of parameters larger than $\operatorname{codim} \mathbf{M}$.

\section{Acknowledgements}

We are grateful to Yu. Ilyashenko for encouraging us to work on global bifurcations of planar vector fields and for valuable discussions. 


\section{References}

[1] A. A. Andronov, E. A. Leontovich, I. I. Gordon, and A. G. Mă̌er. Qualitative theory of second-order dynamic systems. New York-Toronto, Ont. and JerusalemLondon: Halsted Press [A division of John Wiley \& Sons] and Israel Program for Scientific Translations, 1973. xxiii +524 . Trans. of Kachestvennaya teoriya dinamicheskih sistem vtorogo poryadka. Russian. M.: Nauka, 1966. 568 pp.

[2] A. A. Andronov, E. A. Leontovich, I. I. Gordon, and A. G. Mă̌er. Theory of bifurcations of dynamic systems on a plane. Russian. New York-Toronto, Ont. and Jerusalem-London: Halsted Press [A division of John Wiley \& Sons] and Israel Program for Scientific Translations, 1973. xiv+482. Trans. of Teoriya bifurkaciy dinamicheskih sistem na ploskosti. Russian. M.: Nauka, 1967. 488 pp.

[3] A. Andronov and L. Pontrjagin. "Systèmes grossiers". In: Dokl. Akad. Nauk. SSSR 14 (1937), pp. 247-251.

[4] V. I. Arnold, V. S. Afrajmovich, Yu. S. Ilyashenko, and L. P. Shilnikov. Dynamical Systems V. Bifurcation Theory and Catastrophe Theory. Ed. by A. V. I. Vol. 5. Encyclopaedia of Mathematical Sciences. Berlin: Springer-Verlag Berlin Heidelberg, 1994, pp. IX, 274. DOI: 10.1007/978-3-642-57884-7. URL: http: //www.springer.com/gp/book/9783540181736.

[5] H. F. DeBaggis. "Dynamical Systems with Stable Structures". In: Contributions to the Theory of Nonlinear Oscillations. Ed. by S. Lefschetz. Vol. II. Annals of Mathematics Studies 29. Princeton University Press, 1952, pp. 37-60. URL: http: //www.jstor.org/stable/j.ctt1bgz9z7.6.

[6] A. V. Dukov. "Bifurcations of the 'heart' polycycle in generic 2-parameter families". Trans. by I. Marshall. In: Transactions of the Moscow Mathematical Society (2018), pp. 209-229. DOI: $10.1090 / \mathrm{mosc} / 284$. Trans. of "Bifurkacii policikla 'serdce' v tipichnyh dvuparametricheskih semeistvah". In: Trudy Moskovskogo Matematicheskogo Obshchestva 79.2 (2018), pp. 247-269. ISSN: 0134-8663.

[7] A. V. Dukov. "Functional invariants in generic semilocal families of vector fields on two-dimensional sphere". In preparation.

[8] A. V. Dukov and Yu. S. Ilyashenko. "Numerical invariants in generic semilocal families of vector fields on two-dimensional sphere". In preparation.

[9] F. Dumortier and R. Roussarie. "On the saddle loop bifurcation". In: Bifurcations of Planar Vector Fields (Luminy, France, Sept. 18-22, 1989). Ed. by J.-P. Françoise and R. Roussarie. Vol. 1455. Lecture Notes in Mathematics. Berlin, Heidelberg: Springer, 1990, pp. 44-73. ISBN: 978-3-540-46722-9. DOI: 10.1007/BFb0085390.

[10] T. I. Golenishcheva-Kutuzova and V. A. Kleptsyn. "Convergence of the Krylov-Bogolyubov Procedure in Bowan's Example". In: Math. Notes 82.5 (2007), pp. 608-618. DOI: 10.1134/S0001434607110041.

[11] N. B. Goncharuk and Yu. S. Ilyashenko. Large bifurcation supports. Apr. 2018. arXiv: 1804.04596. 
[12] N. Goncharuk and Yu. S. Ilyashenko. "Equivalence relations in bifurcations of planar vector fields". In preparation.

[13] N. Goncharuk, Yu. S. Ilyashenko, and N. Solodovnikov. Global bifurcations in generic one-parameter families with a parabolic cycle on $S^{2}$. To appear in Moscow Math. Jorunal. arXiv: 1707.09779 [math.DS].

[14] N. Goncharuk and Yu. G. Kudryashov. "Bifurcations of planar vector fields: new examples of families with functional invariants". In preparation.

[15] Yu. S. Ilyashenko. "Towards the General Theory of Global Planar Bifurcations". In: Mathematical Sciences with Multidisciplinary Applications. In Honor of Professor Christiane Rousseau. And In Recognition of the Mathematics for Planet Earth Initiative. Ed. by B. Toni. Vol. 157. Springer Proceedings in Mathematics \& Statistics. Cham: Springer, 2016. ISBN: 978-3-319-31321-4. DOI: 10.1007/9783-319-31323-8_13.

[16] Yu. S. Ilyashenko, Yu. G. Kudryashov, and I. Schurov. "Global bifurcations in the two-sphere: a new perspective". In: Inventiones mathematicae 213.2 (Aug. 2018), pp. 461-506. ISSN: 0020-9910. DOI: 10.1007/s00222-018-0793-1. arXiv: 1506:06797. URL: http://rdcu.be/IqCp.

[17] Yu. S. Ilyashenko and N. Solodovnikov. "Global Bifurcations in Generic OneParameter Families with a Separatrix Loop on $S^{2}$. In: Moscow Mathematical Journal 18.1 (January-March 2018), pp. 93-115. ISSN: 1609-4514. URL: http: //www . mathjournals.org/mmj/2018-018-001/2018-018-001-005.html.

[18] Yu. G. Kudryashov and N. Goncharuk. Bifurcations of the polycycle "tears of the heart". multiple numerical invariants. to appear in Moscow Math. J. arXiv: 1808.07459 [math.DS]

[19] Yu. Kuznetsov. Elements of Applied Bifurcation Theory. 3rd ed. Vol. 112. Applied Mathematical Sciences. New York: Springer-Verlag, 2004. XXII, 632. ISBN: 978-14757-3978-7. DOI: 10.1007/978-1-4757-3978-7.

[20] I. P. Malta and J. Palis. "Families of vector fields with finite modulus of stability". In: Dynamical Systems and Turbulence. Proceedings of the Symposium (University of Warwick, 1979-1980). Ed. by D. Rand and L.-S. Young. Vol. 898. Lecture Notes in Mathematics. Berlin, Heidelberg: Springer, 1981, pp. 212-229. ISBN: 978-3-54011171-9. DOI: 10.1007/BFb0091915.

[21] M. M. Peixoto. "On Structural Stability". In: Annals of Mathematics. Second Series 69.1 (Jan. 1959), pp. 199-222. DOI: 10.2307/1970100. URL: http://www. jstor.org/stable/1970100.

[22] M. M. Peixoto. "Structural stability on two-dimensional manifolds". In: Topology 1.2 (Apr. 1, 1962), pp. 101-120. ISSN: 0040-9383. DOI: $10.1016 / 0040-$ 9383(65) 90018-2. URL: http: //www . sciencedirect .com/science/article/ pii/0040938365900182 (visited on 06/20/2017). 
[23] M. M. Peixoto. "Structural stability on two-dimensional manifolds. A further remark". In: Topology 2.1 (Jan. 1, 1963), pp. 179-180. ISSN: 0040-9383. DOI: 10 . 1016/0040-9383(63)90032-6. URL: http://www.sciencedirect.com/science/ article/pii/0040938363900326 (visited on 06/20/2017).

[24] V. Sh. Roitenberg. "Non-local two-parametric bifurcations of planar vector fields". $\mathrm{PhD}$ thesis. Yaroslavl State Technical University, 2000.

[25] V. Sh. Roitenberg. "On bifurcation of vector fields with a separatrix winding onto a polycycle formed by separatrices of two saddles of different types". In: Almanac of contemporary science and education 7 (2012), pp. 116-121. ISSN: 1993-5552.

[26] R. Roussarie. "Weak and continuous equivalences for families on line diffeomorphisms". English. In: Dynamical systems and bifurcation theory, Proc. Meet. (Rio de Janeiro/Braz. 1985). Pitman Res. Notes Math. Ser. 160. 1987, pp. 377-385.

[27] M. V. Shashkov. "On bifurcation of separatrix contours with two saddles". In: International journal of Bifurcation and Chaos 2.4 (1992), pp. 911-915. DOI: 10. 1142/S0218127492000525. URL: https : / / www . worldscientific . com/toc/ $\mathrm{ijbc/02/04}$.

[28] J. Sotomayor. "Generic one-parameter families of vector fields on two-dimensional manifolds". In: Publications Mathématiques de l'Institut des Hautes Études Scientifiques 43.1 (1974), pp. 5-46. ISSN: 0073-8301, 1618-1913. DOI: $10.1007 / \mathrm{BF} 02684365$.

[29] V. Starichkova. "Global Bifurcations in Generic One-parameter Families on $\mathbb{S}^{2}$ ". In: Regular and Chaotic Dynamics 23.6 (2018), pp. 767-784. ISSN: 1560-3547. DOI: 10.1134/S1560354718060102. 